\title{
Plasmonic Sinks for the Selective Removal of Long-Lived States
}

\author{
Stéphane Kéna-Cohen ${ }^{\dagger *}$, Aeneas Wiener ${ }^{\dagger}$, Yonatan $\operatorname{Sivan}^{\dagger}$, Paul N. Stavrinou ${ }^{\dagger}$, \\ Donal D.C. Bradley ${ }^{\dagger}$, Andrew Horsfield ${ }^{\ddagger}$ and Stefan A. Maier ${ }^{\dagger}$ \\ ${ }^{\dagger}$ Department of Physics and \\ ${ }^{\ddagger}$ Department of Materials, \\ Imperial College London, London, SW7 2AZ \\ United Kingdom \\ *Corresponding author: s.kena-cohen@imperial.ac.uk
}

\begin{abstract}
The use of plasmonic nanostructures for the removal of unwanted long-lived states is investigated. We show that the total decay rate of such a state can be increased by up to four orders of magnitude, as compared to its intrinsic radiative decay rate, while leaving other neighboring optical transitions unaffected. For the specific case of molecular triplet excited states, we show that the use of a "plasmonic sink" has the potential to reduce photobleaching and ground state depletion by at least two orders of magnitude. We consider, in addition, the impact of such structures on the performance of organic semiconductor lasers and show that, under realistic device conditions, plasmonic sinks have the capacity to increase the achievable laser repetition rate by a factor equal to the triplet decay rate enhancement. We conclude by studying the effect of exciton diffusion on the triplet density in the presence of metallic nanoparticles.
\end{abstract}

\section{Keywords}

Plasmons, Sinks, Nanoshells, Purcell Effect, Quenching, Triplets, Organic Lasers, Photobleaching, Non-radiative decay 
Plasmonic nanoparticles and antennas have demonstrated significant potential for enhancing the luminescence of adjacent emitters. ${ }^{1-6}$ The mechanisms for enhancement, however, can be varied and the origin of the increased brightness difficult to unravel. For example, the nanostructure can serve to increase the local electric field at the excitation wavelength resulting in an increased excitation rate $^{7-9}$ or equivalently, absorption efficiency of the emitter. In addition, the scattering cross-section of the particle or radiation pattern of the antenna can lead to a higher collection or out-coupling efficiency for the emitted light. ${ }^{2,}{ }^{10}$ Finally, and most importantly for this work, the increased photonic density of states at the emission wavelength can result, via the Purcell effect, in an increased radiative decay rate, $\Gamma_{R}$, for the emitter. ${ }^{1-2,8-10}$ This effect, however, is typically concomitant with an unwanted and stronger increase in the non-radiative decay rate, $\Gamma_{N R}$, due to Joule heating of the metal. ${ }^{9-10}$ In the usual case where $\Gamma_{N R} \gg \Gamma_{R}$, increases in quantum yield, $\phi_{F}$, are only possible for emitters with very low intrinsic yields $\phi_{0}<\Gamma_{R} / \Gamma_{N R} \cdot{ }^{11}$ In this work, we show that instead of being detrimental, the high non-radiative decay rate typical of emitters in close proximity to plasmonic structures can be exploited to selectively remove unwanted excited states in a number of physical systems.

In molecular systems, for example, the build-up of large triplet state populations prevents the efficient excitation of organic molecules with high repetition rates or continuous sources, ${ }^{12-13}$ and can be a significant source of photobleaching. ${ }^{14-17}$ Indeed, Hale et al. first proposed and demonstrated the possibility of using plasmonic nanoparticles to increase the photostability of organic thin films. ${ }^{18}$ In a later section we show that in that case, the effect fits into the "diffusive regime", which has several 
advantages, but where resonant effects play a secondary role. In inorganic semiconductors, plasmonic "sinks" could be used to selectively reduce the lifetime of recombination centers ${ }^{19}$ and defect-bound excitons ${ }^{20}$ or to quench the long-lived surface states that are the source of unwanted "blinking" in colloidal quantum dots. ${ }^{21}$ We will show that if the unwanted state possesses a non-zero transition dipole moment to a lowerlying state, its decay rate can be selectively increased by up to four orders of magnitude. This is a significant departure from previous work on plasmonic fluorescence enhancement where the radiative decay rate from a useful transition is enhanced. Here we focus on resonantly enhancing the total decay rate of an unwanted transition, while leaving the useful transition unaffected. This allows us to use the high $\Gamma_{N R}$ typical of plasmonic structures, often considered a detriment, to our advantage.

The emphasis of this study will be on the selective removal of triplet excitons from fluorescent molecules under high excitation rates, although the same principles hold equally for other systems. In particular, we will show that a modest enhancement of the total decay rate, $\Gamma_{T}=\Gamma_{R}+\Gamma_{N R}$, at the triplet energy, can increase the dye photostability by more than two orders of magnitude due to reduced ground state depletion and photobleaching. We consider both the case where stimulated emission is absent, mimicking the conditions observed in confocal scanning microscopy, ${ }^{12}$ fluorescence lifetime imaging and organic light-emitting diodes (OLEDs), ${ }^{13,22}$ as well as the case where it is present such as in dye lasers ${ }^{23}$ or optically pumped organic semiconductor lasers (OSL). ${ }^{24-29}$ In the latter, we find that although the short-time dynamics are left mostly unaffected, the achievable OSL repetition rate is increased by a factor $\Gamma_{T}$. 


\section{RESULTS AND DISCUSSION}

\section{Plasmonic sink model}

As a model plasmonic sink, we consider spherical silica core/Au shell nanoparticles with, for simplicity, a fluorescent dye positioned a distance $d$ away from the nanoparticle surface. Such positioning can be achieved either through chemical means or via a dielectric spacer layer. ${ }^{30}$ Specific experimental realizations and the consequences of spatial averaging will be considered in the last section. While far from exhaustive, the choice of core/shell particles as a plasmonic sink is based on the well-established synthesis of nanoshells, the narrow plasmonic resonance of these particles and the ease with which the resonance spectral position can be tuned within the visible and near infrared. $^{18,31-33}$ Moreover, the large local density of states on resonance can lead to enhancements of the radiative and non-radiative decay rates of nearby emitters by more than 3 and 4 orders of magnitude, respectively.

In the weak coupling regime, the total decay rate enhancement, $\Gamma_{T}$, of an emitter placed in an inhomogeneous environment can be related to the rate of energy dissipation, $P_{T}$, experienced by a classical electric point dipole: ${ }^{34} \Gamma_{T}=P_{T} / P_{0}$, where $P_{0}$ is the power radiated by the dipole in a homogeneous environment. The electromagnetic fields due to a dipole radiating a distance $d$ from a metallic core/shell particle with inner radius $r_{a}$ and outer radius $r_{b}$, with a dielectric core of refractive index $n_{\mathrm{c}}$, embedded in a dielectric environment with refractive index $n_{b g}$, are obtained by imposing appropriate boundary conditions at the surfaces of the particle (see Methods). This formalism enables us to calculate the enhancement of the rate at which energy leaves a dipole antenna system through radiative $\left(\Gamma_{\mathrm{R}}=P_{R} / P_{0}\right)$, where $P_{R}=P_{T}-P_{N R}$, and through non-radiative $\left(\Gamma_{\mathrm{NR}}=\right.$ 
$\left.P_{N R} / P_{0}\right)$ pathways. To demonstrate the tunability of core/shell particles, the spectral dependence of $\Gamma_{R}$ and $\Gamma_{N R}$ is shown in Fig. 1a, for a horizontal dipole located $d=15 \mathrm{~nm}$ from a Au nanoshell ${ }^{35}$ of outer radius $r_{b}=11 \mathrm{~nm}\left(n_{c}=1.5\right.$ and $\left.n_{b g}=1.7\right)$.

For the particles to be well suited as excitation sinks, they must not quench the useful emission. From Fig. 1a, we see that for this to be the case, the emission needs to be shifted from the resonance position by at least $50 \mathrm{~nm}$. It is also clear from the figure that, on resonance, the decay-rate enhancement is mostly non-radiative in nature. Figure $1 \mathrm{~b}$ shows the spectral dependence of $\Gamma_{\mathrm{T}}$ as a function of distance between the dipole and outer nanoshell surface. We find that for dipoles located within $5 \mathrm{~nm}$, the decay rate is enhanced by more than $10^{4}$, but that the nanoshell is also effective in enhancing the decay rate of dipoles located as much as $d=50 \mathrm{~nm}$ away. The inset shows the quantum efficiency in proximity to the nanoshell of an emitter with unity internal quantum efficiency $\left(\phi_{0}=100 \%\right)$, namely $\Gamma_{R} /\left(\Gamma_{R}+\Gamma_{N R}\right)$. We find that the off-resonance efficiency can be left relatively unaffected for distances $d>20 \mathrm{~nm}$, while simultaneously maintaining a large decay rate enhancement for the unwanted transition (on resonance).

\section{Organic molecules in the absence of stimulated emission}

As an example application, we consider the effect that nanoshells can have on the triplet states that exist in organic molecules. In the lowest order approximation, radiative transitions from the triplet-state (total spin $S=1$ ) to the singlet $(S=0)$ ground state are forbidden. ${ }^{36-37}$ As a result, the "dark" triplet state is the source of blinking in singlemolecule fluorescence, ${ }^{38}$ annihilation mechanisms in lasers ${ }^{23-26}$ and a significant source of photobleaching. ${ }^{12,14-15}$ Spin-orbit coupling, however, mixes the singlet and triplet 
states imparting the transition with a finite transition dipole moment. In the absence of extraneous quenching mechanisms, such as are provided by impurities or energy transfer to the triplet ground state of molecular oxygen, ${ }^{37,39}$ triplet lifetimes are typically on the order of $10^{-4}-1 \mathrm{s.}^{36-37}$

The molecular energy level structure that we are considering is depicted in Fig. 2. Upon non-resonant optical excitation, high energy electronically excited states, $S_{n}$, are generated within the singlet manifold. These excitations rapidly relax via internal conversion (I.C.) and vibronic relaxation to the bottom of $S_{l}$, the first excited singlet state, with typical rates $k_{I C} \sim 10^{11}-10^{13} \mathrm{~s}^{-1}$. Here, we ignore this fast initial relaxation and treat both the singlet and triplet states as effective two-level systems. To simplify the discussion, we also ignore non-radiative internal conversion from $S_{l}$ to $S_{0}$ and intersystem crossing from $T_{1}$ to $S_{0}$. As a result, singlet excitations are only lost via radiation and intersystem crossing to the triplet state (Ermolaev's rule ${ }^{37}$ ) and triplets are only lost via radiation. These assumptions are clearly molecule and environment-dependent, ${ }^{40}$ but they allow us to directly express the singlet and triplet lifetimes as $\tau_{S} / \Gamma_{T}\left(E_{S}\right)$ and $\tau_{T} / \Gamma_{T}\left(E_{T}\right)$, where $\tau_{S}, \tau_{T}$ are the bare singlet and triplet lifetimes and the decay rate is evaluated at their respective energies, $E_{S}$ and $E_{T}$. In proximity to the plasmonic sink, however, the importance of these assumptions is reduced since transition-dipole induced transitions can be made to dominate over other radiationless pathways.

Following $\mathrm{Xu}$ et al., we neglect fluorescence decay within the duration of the excitation pulse and define an excitation probability per molecule, $a$, which is related to the absorption cross-section, $\sigma_{a}$, and the incident irradiance $I(t):{ }^{17}$

$$
a=1-\exp \left(-\sigma_{a} \int I(t) d t\right)
$$


where the irradiance is integrated over a single pulse. In this picture, the number of photons emitted via fluorescence after a single pulse is given by: $N_{F L}=a \phi_{F} N_{0}$, where $N_{0}$ is the total number of molecules and $\phi_{F}$ is the quantum efficiency.

We first consider the effect of reducing the triplet lifetime by examining the change in fluorescence intensity as a function of the pulse number. The emitter intersystem crossing yield, $\phi_{I S C}$, is defined such that after each pulse $a \phi_{I S C} N_{g}(n)$ of the excitations are funneled to the triplet state, where $N_{g}(n)$ is the ground state population immediately before the $n$-th exciting pulse. In the absence of annihilation mechanisms, the ground state population can be calculated by assuming that all singlets from the previous pulse have decayed. ${ }^{17}$ We find:

$$
N_{g}(n)=N_{0} \frac{1-e^{-\Delta t / \tau_{T}}+a \phi_{I S C}\left(1-a \phi_{I S C}\right)^{n} e^{-(n+1) \Delta t / \tau_{T}}}{1-e^{-\Delta t / \tau_{T}}\left(1-a \phi_{I S C}\right)}
$$

where $\Delta t=1 / f$, is the spacing between pulses, $f$ is the repetition rate and $\tau_{T}$ is the triplet lifetime.

The resulting fluorescence intensity is shown using dashed lines in Fig. 3a for the case $\phi_{I S C}=0.01, f=80 \mathrm{MHz}, \tau_{T}=100 \mu \mathrm{s}$. We find that for large excitation probabilities, $a$, the fluorescence intensity decreases rapidly after $>10^{3}$ pulses due to a depletion of the ground-state. The molecules are "trapped" in the dark triplet state. It is clear from the results shown in Fig. 3a that significant ground-state depletion occurs only for $a \sim 1$. These, however, are precisely the conditions that are often used in single or few-molecule spectroscopy where a high irradiance is needed to obtain an observable fluorescence signal. The solid-lines in Fig. 3a show the fluorescence intensity in the presence of nanoshells designed to increase the decay rate at a triplet wavelength, $\lambda_{T}=750 \mathrm{~nm}$, 
chosen to correspond to the results shown in from Fig. 1b. We use a decay rate enhancement $\Gamma_{T}=100$, which is achieved at a distance $d=20 \mathrm{~nm}$, resulting in an effective lifetime $\tau_{T} / \Gamma_{\mathrm{T}}=1 \mu$ s and a quantum efficiency reduction of less than $50 \%$ for fluorescence wavelengths $\lambda_{S}<550 \mathrm{~nm}$. We find that even for $a=1$, the steady-state value, which can be shown to be $N_{g}(n)=\left(1+a \phi_{I S C} \tau_{T} / \Delta t\right)^{-1}$ for $\Delta \mathrm{t} / \tau_{\mathrm{T}}<<1$, remains greater than $50 \%$ at $80 \mathrm{MHz}$. It can also be shown that steady-state is reached after fewer pulses, $n \sim \tau_{\mathrm{T}} / \Gamma_{\mathrm{T}} \Delta \mathrm{t}$, for the reduced triplet lifetime. ${ }^{17}$

In addition to reducing the ground state depletion, the increased triplet decay rate can significantly improve the dye photostability. Indeed, triplets are well-known to be a significant pathway for photobleaching. For example, excitations in the $T_{n}$ manifold can be photoionized, resulting in the formation of free radicals, they can react with ionic species, or lead to irreversible photoproducts due to the formation of singlet oxygen. ${ }^{14-15}$, ${ }^{37}$ Indeed, excitations into high-lying triplet states have been identified as the main pathway for bleaching in two-step photolysis. ${ }^{15}$ The reasons for this are two-fold: the triplet-triplet absorption cross-section is often larger and spectrally broader than that for singlet-singlet absorption and, at the repetition rates used under typical experimental conditions for imaging, most excited singlets have relaxed to the ground state prior to the arrival of the next pulse, while in contrast most triplets are still in the excited state. Note that Enderlein has previously considered potential increases in photostability due to enhancements of the molecular fluorescence rate in the proximity of metallic structures. ${ }^{41-42}$ For the triplet sinks discussed in the previous section, the fluorescence rate enhancement is negligible and we ignore this effect. 
If we assume that the probabilities for photodegradation from the singlet and triplet state are $Q_{S b}$ and $Q_{T b}$, respectively, and ignore photobleaching reactions from higher-lying states, we can derive a set of coupled difference equations for the triplet and ground-state populations immediately before the $n$-th pulse:

$$
\begin{gathered}
N_{T}(n)=e^{-\Delta t / \tau_{T}}\left[N_{T}(n-1)+a \phi_{I S C}\left(1-Q_{b T}\right) N_{g}(n-1)\right] \\
N_{g}(n)=\left[1-a\left(Q_{b}+\phi_{I S C} e^{-\Delta t \tau_{T}}\right)\right] N_{g}(n-1)+N_{T}(n-1)\left(1-e^{-\Delta t / \tau_{T}}\right)
\end{gathered}
$$

where we have defined $Q_{b}=\phi_{I S C} Q_{T b}+\left(1-\phi_{I S C}\right) Q_{S b}$. These equations can easily be solved analytically. To our knowledge, there have been no reported measurements of $Q_{S b}$ and $Q_{T b}$. The overall photobleaching yield $Q_{b}$, however, has been measured for a number of dyes. ${ }^{15,43-44}$ The dashed lines in Fig. 3b show the relative fluorescence yield obtained from the solution of Eqs. (3-4) by assuming a fluorescence lifetime $\tau_{\mathrm{S}}=5 \mathrm{~ns}$ and equal reactivities for bleaching $k_{S b}=k_{T b}=100 \mathrm{~s}^{-1}$, such that $Q_{S b}=k_{S b} \tau_{S}$ and $Q_{T b}=k_{T b} \tau_{T}$, from the singlet and triplet states, respectively. The "kink" observed in the fluorescence marks the transition between fluorescence loss dominated by ground state depletion and that dominated by irreversible photobleaching. Photobleaching is clearly the dominant loss mechanism for low excitation probabilities. The fluorescence intensity as a function of pulse number, in the presence of the plasmonic sink, is shown as solid lines in Fig. $3 \mathrm{~b}$. Again, assuming $\Gamma_{T}=100$, it can be seen that the dye photostability can be increased, in some cases, by $>2$ orders of magnitude. If the triplet lifetime is reduced further, however, such that $\phi_{I S C} \tau_{T}<\tau_{S}$, photobleaching via the singlet state becomes the dominant pathway.

\section{Organic molecules in the presence of stimulated emission}


The presence of triplets is also well known to be a limiting factor for the realization of continuous wave $(\mathrm{CW})$ dye lasers. In many cases, triplet-triplet absorption (TTA) due to the excitation of triplets, $T_{l}$, to higher-lying states $T_{n}$ is responsible for increasing the losses under $\mathrm{CW}$ operation. Dye jets and triplet quenchers such as oxygen or cyclooctatetraene are commonly used to overcome TTA. ${ }^{16,}{ }^{45}$ In optically pumped organic semiconductor lasers (OSLs), the solid-state analog of dye lasers, triplets also limit $\mathrm{CW}$ and quasi-CW operation. ${ }^{26-27}$ This can occur both through TTA and singlettriplet annihilation (STA). In the latter, energy is lost when a singlet transfers its excitation to a neighboring triplet, thus exciting it to a higher energy level, $T_{n}$, which subsequently relaxes. For example, under $\mathrm{CW}$ excitation, lasing in OSLs was shown to persist for $<150 \mathrm{~ns}$ even at pump powers twice that of threshold. ${ }^{26}$ In this section, we model the effect of the plasmonic sink on an OSL (see Methods) using material parameters, given in Table 1, that are typical of low molecular weight and polymer lasers. $^{25-26}$

The transient output intensity of the laser, for excitation with a square pulse at a pump rate $R=1.5 R_{t h} 0$, where $R_{t h 0}$ is the laser threshold in the absence of triplets, is shown as the dashed lines in Fig. 4 for the case of STA or TTA for two different values of $\phi_{I S C}$. Relaxation oscillations are present at short times, but the most salient feature is the rapid quenching of lasing action when either STA or TTA is present. The solid lines show the transient behaviour in the presence of the plasmonic sinks. It can be seen from Fig. 4 that in this case, the laser pulse duration is only slightly increased. Indeed, from Eq. (9) (see Methods) it is clear that for $\Delta \mathrm{t} / \tau_{\mathrm{T}}<<1$, the triplet density is simply the integral of $\phi_{I S C} R(t)$. In fact, in the single pulse regime, lasing can always be achieved for pump rates 
above $R_{t h 0}$, provided that the excitation pulse is made short enough. The situation, however, changes drastically in the multi-pulse regime.

Following our previous analysis and again assuming that the singlet population has decayed prior to the arrival of each pulse, but neglecting ground-state depletion, we can solve for the steady-state triplet density:

$$
N_{T}=\frac{\kappa e^{-\Delta t / \tau_{T}}}{1-e^{-\Delta t / \tau_{T}}}
$$

with $\kappa \equiv \phi_{I S C} \int R(t) e^{t / \tau_{T}} d t$, where the integral is defined over one pulse duration. The steady-state triplet density is shown in Fig. 5a as a function of repetition rate for different pump rates assuming $20 \mathrm{~ns}$-long square pulses. Also shown in the figure is the corresponding triplet density in the presence of the plasmonic sink. Concomitant with the decrease in lifetime of two orders of magnitude, we find an increase of two orders of magnitude in the achievable repetition rate for a given triplet density. If $\Delta \mathrm{t} / \tau_{\mathrm{T}}<<1$, the repetition rate needed to achieve a given triplet density can be shown to be proportional to $\Gamma_{T}$, the reduction in the triplet lifetime.

This finding also allows us to determine the maximum achievable repetition rate when either STA or TTA is present. Figure $5 \mathrm{~b}$ shows the ratio $R_{t h}^{S T A(T T A)} / R_{t h 0}$, where $R_{t h}^{S T A(T T A)}$ is the excitation rate threshold in the presence of STA (TTA), for varying excitation rates $R$. The endpoints to each curve show the maximum repetition rate that can be achieved for the specified pump power. We find that the reduced triplet density at higher repetition rate for reduced triplet lifetimes directly correlates to an increase in the achievable OSL frequency. 


\section{Design considerations and the diffusive regime}

Until now, we have considered the decay rate enhancement resulting from a dipole with a horizontal orientation located at a fixed distance from the nanoshell. The distance was chosen to achieve a reasonable compromise between the reduction in quantum efficiency and that in triplet lifetime. In practice, the optimal approach depends on the situation at hand. For imaging in solution, ${ }^{46}$ where molecules are subject to diffusion, directly attaching the dye molecule to the outside (or even inside) of the nanoshell is a viable approach. ${ }^{30,47}$ This approach is also suitable for the case of immobile molecules such as those in a lightly doped matrix. Loading the matrix with nanoparticles is also possible and at densities with a surface to surface spacing $>60 \mathrm{~nm}$, near-field coupling effects are negligible. The density, however, must be high enough to effectively quench the triplet state, but not so high as to cause extraneous quenching of the useful emission.

The most advantageous situation is found for the case of neat organic films such as those used in OSLs and OLEDs. In those cases, the excited states are mobile singlet and triplet excitons, which can hop between adjacent molecules. ${ }^{36}$ Due to their long lifetimes, triplet excitons possess diffusion lengths much larger than those of their singlet counterparts. For example, reported triplet diffusion lengths are in the $10^{2}-10^{4} \mathrm{~nm}$ range $^{48-49}$ as compared to singlet diffusion lengths that rarely exceed $30 \mathrm{~nm}^{50}$ The initial demonstration of reduced photobleaching in polymer films by Hale et al. fits into this category. ${ }^{18}$ If the triplet diffusion length is much larger than the effective capture radius of the plasmonic sink, resonant effects play a secondary role and both the singlet and triplet densities can be assumed to vanish at the metal surface. 
To illustrate this effect, we consider a 1D lattice of metal nanoparticles, with spacing $L$. The steady-state singlet, $N_{S}$, and triplet, $N_{T}$, densities are governed by the diffusion equation:

$$
D_{S, T} \frac{d^{2} N_{S, T}}{d x^{2}}-\frac{N_{S, T}}{\tau_{S, T}}+G_{S, T}=0
$$

where the $D_{S, T}$ is the diffusivity, $\tau_{S, T}$ is the lifetime and $G_{S, T}$ is the generation rate of singlets and triplets, as indicated by the subscript. Regardless of their shape, the nanoparticles will result in vanishing surface exciton densities. The solution, for a constant generation rate, is given by:

$$
N_{S, T}(x)=\sum_{n=1,3,5, . .}^{\infty} \frac{4}{n \pi} \frac{G_{S, T}}{D_{S, T}(\pi n / L)^{2}+\tau_{S, T}^{-1}} \sin \left(\frac{\pi n x}{L}\right)
$$

The resulting densities, normalized by the densities in the absence of quenching, $N_{S O, T O}$, are shown in Fig. 6a for varying lattice spacings. The result, which depends only on the singlet and triplet diffusion lengths, $L_{D, S} \equiv \sqrt{D_{S} \tau_{S}}$ and $L_{D, T} \equiv \sqrt{D_{T} \tau_{T}}$, is shown for $L_{D, S}$ $=20 \mathrm{~nm}$ and $L_{D, T}=250 \mathrm{~nm}$. It is clear, both conceptually and from the figure, that an optimum spacing exists which reduces the triplet density, while least affecting singlets. This optimum spacing, $L_{\text {opt }}$, can be found my maximizing the objective function $N_{S} / N_{S 0}-N_{T} / N_{T 0}$ and is given in Fig. 6b. As expected, $L_{o p t}$ is on the order of the triplet diffusion length, but decreases for shorter $L_{D, S}$. As a result, for mobile excitons, the spacing between nanoparticles can be several hundred nanometers and still result in a large quenching efficiency for triplets, while having a negligible impact on the singlet concentration. For higher dimensionality, the capture radius is more important due to the availability of new escape paths, but the results are qualitatively similar. Figures $6 \mathrm{c}$ and 
$6 \mathrm{~d}$ show the singlet and triplet densities obtained using identical parameters and the $2 \mathrm{D}$ optimum spacing $L_{\text {opt }}=150 \mathrm{~nm}$. The extremely low nanoparticle concentration required means that optical losses, which scale as the metal filling fraction, can be kept negligible justifying our neglect of additional cavity losses in the analysis of OSL dynamics.

\section{CONCLUSION}

We have investigated the use of metallic nanoparticles as plasmonic sinks for unwanted excitations. We take advantage of the large non-radiative decay rate and spectral selection that can be achieved using localized plasmonic resonances to quench the unwanted states, while leaving other states unaffected. This approach is viable if the unwanted state possesses a non-zero transition dipole moment and it results in a reduced

lifetime for that state: $\tau_{0}\left[\Gamma_{T} \phi_{T}+\left(1-\phi_{T}\right)\right]^{-1}$ where $\tau_{0}$ is its bare lifetime, and $\phi_{T}$ its intrinsic quantum efficiency. We have shown that for the particular case of triplet excitations, a modest reduction in the triplet lifetime $\Gamma_{T}=100$ can result in a photostability increase of more than two orders of magnitude and an increase of the achievable repetition rate for OSLs concomitant with the reduction in lifetime. Due to the long diffusion lengths typical of triplet excitons, this scheme is particularly advantageous for the case of organic films, as demonstrated by Hale et al., where the quenching achievable by a single nanoparticle can be made extremely long-range, while leaving singlets unaffected. 


\section{METHODS}

\section{Dipole emission in the presence of nanoshells}

To calculate the decay rate enhancements we follow the aproach by Ruppin, ${ }^{51}$ who showed that for a solid metal sphere, the electromagnetic fields may be expanded in a series of spherical vector functions, to express the total power $P_{T}$ emitted by the dipole and $P_{N R}$, the power lost non-radiatively due to Joule heating in the metal, in terms of the Mie scattering coefficients. For a nanoshell, the solution is expressed in terms of the Mie scattering coefficients of the nanoshell, ${ }^{52}$ but is otherwise unchanged. ${ }^{53}$ We have established the validity of this formalism through finite difference time domain (FDTD) calculations.

\section{Organic semiconductor laser model}

The rate equations determining the singlet, triplet and photon densities, $S$, in the presence of stimulated emission are: $:^{23,26}$

$$
\begin{gathered}
\frac{d N_{S}}{d t}=\left(1-\phi_{I S C}\right) R(t)-\frac{N_{S}}{\tau_{S}}-k_{S T} N_{S} N_{T}-c N_{S} \sigma_{e} S \\
\frac{d N_{T}}{d t}=\phi_{I S C} R(t)-\frac{N_{T}}{\tau_{T}} \\
\frac{d S}{d t}=c\left(N_{S} \sigma_{e}-\alpha_{c a v}-\sigma_{T T} N_{T}\right) S+\beta_{s p} \frac{N_{S}}{\tau_{S}}
\end{gathered}
$$

where $\sigma_{e}$ is the stimulated emission cross-section, $\alpha_{c a v}$ is the cavity loss, $k_{S T}$ is the STA rate, $\sigma_{T T}$ is the TTA cross-section, $\beta_{s p}$ is the spontaneous emission factor and $c$ is the speed of light in the medium. The confinement factor is assumed to be unity so that the TTA modal loss can be directly added to the cavity loss. Note that a key assumption 
when discussing triplet behaviour in the presence of stimulated emission is whether triplets are generated with a fixed yield $\phi_{I S C}$ or fixed rate constant $k_{I S C}$. The high densities of triplets observed under optical excitation in dye lasers, guest-host systems with rapid Forster transfer rates and using ultrafast spectroscopy suggest that for excitation energies

above that of the singlet, the dynamics are better described using a fixed yield. ${ }^{26,28,54-56}$ In contrast, the assumption of a fixed $k_{I S C}$ would lead to a nearly complete depletion of the triplet state under stimulated emission.

\section{Acknowledgements}

The authors acknowledge the UK Engineering and Physical Sciences Research Council (Active Plasmonics) for funding. S.K.C. acknowledges financial support from the Fonds Québécois de Recherche sur la Nature et les Technologies and Y.S. acknowledges support from the Royal Society International Newton Fellowship. 


\section{REFERENCES}

1. Anger, P.; Bharadwaj, P.; Novotny, L., Enhancement and Quenching of SingleMolecule Fluorescence. Phys. Rev. Lett. 2006, 96, 113002.

2. Bardhan, R.; Grady, N. K.; Cole, J. R.; Joshi, A.; Halas, N. J., Fluorescence Enhancement by Au Nanostructures: Nanoshells and Nanorods. Acs Nano 2009, 3, 744752.

3. Giannini, V.; Fernandez-Dominguez, A. I.; Heck, S. C.; Maier, S. A., Plasmonic Nanoantennas: Fundamentals and Their Use in Controlling the Radiative Properties of Nanoemitters. Chem. Rev. 2011, 111, 3888-3912.

4. Kuhn, S.; Hakanson, U.; Rogobete, L.; Sandoghdar, V., Enhancement of SingleMolecule Fluorescence Using a Gold Nanoparticle as an Optical Nanoantenna. Phys. Rev. Lett. 2006, 97, 017402.

5. Song, J. H.; Atay, T.; Shi, S. F.; Urabe, H.; Nurmikko, A. V., Large Enhancement of Fluorescence Efficiency from Cdse/Zns Quantum Dots Induced by Resonant Coupling to Spatially Controlled Surface Plasmons. Nano Lett. 2005, 5, 1557-1561.

6. Tam, F.; Goodrich, G. P.; Johnson, B. R.; Halas, N. J., Plasmonic Enhancement of Molecular Fluorescence. Nano Lett. 2007, 7, 496-501.

7. Aouani, H.; Itzhakov, S.; Gachet, D.; Devaux, E.; Ebbesen, T. W.; Rigneault, H.;

Oron, D.; Wenger, J., Colloidal Quantum Dots as Probes of Excitation Field Enhancement in Photonic Antennas. Acs Nano 2010, 4, 4571-4578. 
8. Giannini, V.; Muskens, O. L.; Rivas, J. G.; Sanchez-Gil, J. A., Electrodynamic Calculations of Spontaneous Emission Coupled to Metal Nanostructures of Arbitrary Shape: Nanoantenna-Enhanced Fluorescence. J Opt Soc Am B 2009, 26, 1569-1577.

9. Rogobete, L.; Kaminski, F.; Agio, M.; Sandoghdar, V., Design of Plasmonic Nanoantennae for Enhancing Spontaneous Emission. Opt Lett 2007, 32, 1623-1625.

10. Vandenbem, C.; Brayer, D.; Froufe-Perez, L. S.; Carminati, R., Controlling the Quantum Yield of a Dipole Emitter with Coupled Plasmonic Modes. Phys. Rev. B 2010, $81,085444$.

11. Giannini, V.; Fernandez-Dominguez, A. I.; Sonnefraud, Y.; Roschuk, T.; Fernandez-Garcia, R.; Maier, S. A., Controlling Light Localization and Light-Matter Interactions with Nanoplasmonics. Small 2010, 6, 2498-2507.

12. Donnert, G.; Eggeling, C.; Hell, S. W., Major Signal Increase in Fluorescence Microscopy through Dark-State Relaxation. Nat. Methods 2007, 4, 81-86.

13. Zhang, Y. F.; Whited, M.; Thompson, M. E.; Forrest, S. R., Singlet-Triplet Quenching in High Intensity Fluorescent Organic Light Emitting Diodes. Chem. Phys. Lett. 2010, 495, 161-165.

14. Eggeling, C.; Volkmer, A.; Seidel, C. A. M., Molecular Photobleaching Kinetics of Rhodamine $6 \mathrm{~g}$ by One- and Two-Photon Induced Confocal Fluorescence Microscopy. Chemphyschem 2005, 6, 791-804.

15. Eggeling, C.; Widengren, J.; Rigler, R.; Seidel, C. A. M., Photobleaching of Fluorescent Dyes under Conditions Used for Single-Molecule Detection: Evidence of Two-Step Photolysis. Anal Chem 1998, 70, 2651-2659. 
16. Pappalardo, R.; Samelson, H.; Lempicki, A., Long Pulse Laser Emission from Rhodamine 6-G Using Cyclooctatetraene. Appl. Phys. Lett. 1970, 16, 267.

17. Xu, C.; Webb, W., Multiphoton Excitation of Molecular Fluorophores and Nonlinear Laser Microscopy

Topics in Fluorescence Spectroscopy. Lakowicz, J., Ed. Springer US: 2002; Vol. 5, pp 471-540.

18. Hale, G. D.; Jackson, J. B.; Shmakova, O. E.; Lee, T. R.; Halas, N. J., Enhancing the Active Lifetime of Luminescent Semiconducting Polymers Via Doping with Metal Nanoshells. Appl. Phys. Lett. 2001, 78, 1502-1504.

19. Queisser, H. J., Defects in Semiconductors: Some Fatal, Some Vital. Science 1998, 281, 945-950.

20. Charbonneau, S.; Steiner, T.; Thewalt, M. L. W., Photoluminescence Decay Times of the Defect-Induced Bound-Exciton Lines in Gaas Grown by Molecular-Beam Epitaxy. Phys. Rev. B 1990, 41, 2861.

21. Mahler, B.; Spinicelli, P.; Buil, S.; Quelin, X.; Hermier, J. P.; Dubertret, B., Towards Non-Blinking Colloidal Quantum Dots. Nat Mater 2008, 7, 659-64.

22. Baldo, M. A.; Segal, M.; Holmes, R. J.; Forrest, S. R.; Soos, Z. G., Excitonic Singlet-Triplet Ratios in Molecular and Polymeric Organic Materials. Phys. Rev. B 2003, $68,075211$.

23. Peterson, O. G.; Webb, J. P.; Mccolgin, W. C.; Eberly, J. H., Organic Dye Laser Threshold. J. Appl. Phys. 1971, 42, 1917.

24. Baldo, M. A.; Holmes, R. J.; Forrest, S. R., Prospects for Electrically Pumped Organic Lasers. Phys. Rev. B 2002, 66, 035321. 
25. Gartner, C.; Karnutsch, C.; Lemmer, U.; Pflumm, C., The Influence of Annihilation Processes on the Threshold Current Density of Organic Laser Diodes. $J$. Appl. Phys. 2007, 101, 023107.

26. Giebink, N. C.; Forrest, S. R., Temporal Response of Optically Pumped Organic Semiconductor Lasers and Its Implication for Reaching Threshold under Electrical Excitation. Phys. Rev. B 2009, 79, 073302.

27. Rabe, T., et al., Quasi-Continuous-Wave Operation of an Organic Thin-Film Distributed Feedback Laser. Appl. Phys. Lett. 2006, 89.

28. Reufer, M.; Lupton, J. M.; Scherf, U., Stimulated Emission Depletion of Triplet Excitons in a Phosphorescent Organic Laser. Appl. Phys. Lett. 2006, 89, 141111.

29. Xia, R. D.; Heliotis, G.; Hou, Y. B.; Bradley, D. D. C., Fluorene-Based Conjugated Polymer Optical Gain Media. Org Electron 2003, 4, 165-177.

30. Thomas, K. G.; Kamat, P. V., Chrompophore Functionalized Metal Nanoparticles. Abstr Pap Am Chem S 2004, 227, U883-U883.

31. Averitt, R. D.; Westcott, S. L.; Halas, N. J., Linear Optical Properties of Gold Nanoshells. J Opt Soc Am B 1999, 16, 1824-1832.

32. Prodan, E.; Radloff, C.; Halas, N. J.; Nordlander, P., A Hybridization Model for the Plasmon Response of Complex Nanostructures. Science 2003, 302, 419-422.

33. Sun, Y. G.; Xia, Y. N., Gold and Silver Nanoparticles: A Class of Chromophores with Colors Tunable in the Range from 400 to 750 Nm. Analyst 2003, 128, 686-691.

34. Novotny, L.; Hecht, B., Principles of Nano-Optics. Cambridge University Press: Cambridge ; New York, 2006; p 539 p. 
35. Palik, E. D., Handbook of Optical Constants of Solids. Academic Press: Orlando, $1985 ; \mathrm{p} 804$ p.

36. Pope, M.; Swenberg, C. E., Electronic Processes in Organic Crystals and Polymers. 2nd ed.; Oxford University Press: New York, 1999; p 1328 p.

37. Turro, N. J., Modern Molecular Photochemistry. Benjamin/Cummings Pub. Co.: Menlo Park, Calif., 1978; p 628 p.

38. Basche, T.; Kummer, S.; Brauchle, C., Direct Spectroscopic Observation of Quantum Jumps of a Single Molecule. Nature 1995, 373, 132-134.

39. Hubner, C. G.; Renn, A.; Renge, I.; Wild, U. P., Direct Observation of the Triplet Lifetime Quenching of Single Dye Molecules by Molecular Oxygen. J. Chem. Phys. 2001, 115, 9619-9622.

40. In the presence of extraneous quenching mechanisms, such as those mentioned in the previous paragraph, the modified triplet lifetime is given by: $\tau_{T}\left[\Gamma_{T} \phi_{P}+\left(1-\phi_{P}\right)\right]^{-1}$, where $\phi_{P}$ is the phosphorescence yield.

41. Enderlein, J., Single-Molecule Fluorescence near a Metal Layer. Chem. Phys. 1999, 247, 1-9.

42. Enderlein, J., Spectral Properties of a Fluorescing Molecule within a Spherical Metallic Nanocavity. Phys. Chem. Chem. Phys. 2002, 4, 2780-2786.

43. Ippen, E. P.; Shank, C. V.; Dienes, A., Rapid Photobleaching of Organic Laser Dyes in Continuously Operated Devices. IEEE J. Quantum. Electron. 1971, Qe 7, 178.

44. Bout, D. A. V.; Deschenes, L. A., Single Molecule Photobleaching: Increasing Photon Yield and Survival Time through Suppression of Two-Step Photolysis. Chem. Phys. Lett. 2002, 365, 387-395. 
45. Schols, S.; Kadashchuk, A.; Heremans, P.; Helfer, A.; Scherf, U., Triplet Excitation Scavenging in Films of Conjugated Polymers. Chemphyschem 2009, 10, 10711076.

46. In organic solvents at room temperature, the phosphorescence yield is typically quite low. If that is the case, $\Gamma_{\mathrm{T}}$ needs to be correspondingly large to affect the overall triplet decay rate.

47. Zhang, J.; Gryczynski, I.; Gryczynski, Z.; Lakowicz, J. R., Dye-Labeled Silver Nanoshell-Bright Particle. J Phys Chem B 2006, 110, 8986-8991.

48. Giebink, N. C.; Sun, Y.; Forrest, S. R., Transient Analysis of Triplet Exciton Dynamics in Amorphous Organic Semiconductor Thin Films. Org Electron 2006, 7, 375386.

49. Samiullah, M.; Moghe, D.; Scherf, U.; Guha, S., Diffusion Length of Triplet Excitons in Organic Semiconductors. Phys. Rev. B 2010, 82, 205211.

50. Lunt, R. R.; Giebink, N. C.; Belak, A. A.; Benziger, J. B.; Forrest, S. R., Exciton Diffusion Lengths of Organic Semiconductor Thin Films Measured by Spectrally Resolved Photoluminescence Quenching. J. Appl. Phys. 2009, 105.

51. Ruppin, R., Decay of an Excited Molecule near a Small Metal Sphere. J. Chem. Phys. 1982, 76, 1681-1684.

52. Kerker, M.; Kauffman, L. H.; Farone, W. A., Scattering of Electromagnetic Waves from Two Concentric Spheres When the Outer Shell Has a Variable Refractive Index. Numerical Results. J. Opt. Soc. Am. 1966, 56, 1053-1055.

53. Gibson, J. W.; Johnson, B. R., Density-Matrix Calculation of Surface-Enhanced Raman Scattering for P-Mercaptoaniline on Silver Nanoshells. J. Chem. Phys. 2006, 124. 
54. Gradinaru, C. C.; Kennis, J. T. M.; Papagiannakis, E.; van Stokkum, I. H. M.; Cogdell, R. J.; Fleming, G. R.; Niederman, R. A.; van Grondelle, R., An Unusual Pathway of Excitation Energy Deactivation in Carotenoids: Singlet-to-Triplet Conversion on an Ultrafast Timescale in a Photosynthetic Antenna. Proc Natl Acad Sci U S A 2001, 98, 2364-2369.

55. Lanzani, G.; Cerullo, G.; Zavelani-Rossi, M.; De Silvestri, S.; Comoretto, D.; Musso, G.; Dellepiane, G., Triplet-Exciton Generation Mechanism in a New Soluble (Red-Phase) Polydiacetylene. Phys. Rev. Lett. 2001, 87.

56. Zenz, C.; Cerullo, G.; Lanzani, G.; Graupner, W.; Meghdadi, F.; Leising, G.; De Silvestri, S., Ultrafast Photogeneration Mechanisms of Triplet States in Para-Hexaphenyl. Phys. Rev. B 1999, 59, 14336-14341. 
Table 1: Organic laser material parameters

\begin{tabular}{cc}
\hline Parameter & Value \\
\hline$\sigma_{e}$ & $10^{-16} \mathrm{~cm}^{2}$ \\
$\sigma_{T T}$ & $10^{-17} \mathrm{~cm}^{2}$ \\
$k_{S T}$ & $10^{-10} \mathrm{~cm}^{-3} \mathrm{~s}^{-1}$ \\
$\tau_{S}$ & $5 \mathrm{~ns}$ \\
$\tau_{T}$ & $100 \mu \mathrm{s}$ \\
$\alpha_{\text {cav }}$ & $15 \mathrm{~cm}^{-1}$ \\
$\beta$ & $10^{-4}$ \\
$n$ & 1.7 \\
\hline \hline
\end{tabular}




\section{FIGURE CAPTIONS}

Figure 1: a) Enhancement of the radiative (bottom) and non-radiative (top) decay rates obtained for a horizontal dipole located at a distance $d=15 \mathrm{~nm}$ from a Au nanoshell [ $\left.\mathrm{n}_{\mathrm{c}}=1.5, \mathrm{n}_{\mathrm{bg}}=1.7\right]$ of outer radius $r_{b}=11 \mathrm{~nm}$, for varying values of $r_{a}$, the inner core radius. b) Contour map of the total decay rate enhancement for a nanoshell with $r_{a}=9$ $\mathrm{nm}, r_{b}=11 \mathrm{~nm}$ shown as a function of the distance between the dipole and the outer shell surface. The inset shows the resulting quantum efficiency assuming an emitter with unity internal quantum efficiency.

Figure 2: Schematic diagram showing the energy levels that participate in the photoemission process. Non-resonant excitation brings the molecule to a highly-excited state. This state relaxes to the bottom of the excited singlet state $\left(S_{I}\right)$ by internal conversion (I.C.) and vibronic relaxation. Some fraction of the excitations are funneled to the long-lived triplet state $\left(T_{1}\right)$ by intersystem crossing. Excited singlets and triplets relax to the ground state with respective lifetimes $\tau_{S}$ and $\tau_{T}$. To simplify the discussion we assume that both transitions are radiative in nature. As a result the reduction in triplet lifetime resulting from the presence of the plasmonic sink is simply $\tau_{\mathrm{T}} / \Gamma_{\mathrm{T}}$.

Figure 3: a) Relative fluorescence efficiency given for different values of the excitation probability, $a$, as a function of the number of exciting pulses, for $f=80 \mathrm{MHz}$. It is seen that for high excitation probabilities, the ground state becomes highly depleted. The solid lines show the relative fluorescence efficiency for the reduced triplet lifetime resulting 
from the presence of the plasmonic sink. b) Relative fluorescence efficiency accounting for both ground state depletion and photobleaching with microscopic reactivities $k_{S b}=k_{T b}$ $=100 \mathrm{~s}^{-1}$ from the singlet and triplet states. The solid lines show the strong increase in photostability resulting from the reduced triplet lifetime. For a) and b), the number of photons emitted per pulse is obtained by multiplying the relative fluorescence efficiency by $a \phi_{F} N_{0}$, where $N_{0}$ is the number of molecules.

Figure 4: Output intensity from an OSL, using the parameters given in Table 1, excited with a square pulse of $\mathrm{t}_{\mathrm{R}}=35 \mathrm{~ns}$ rise time, at an excitation rate $R=1.5 R_{t h 0}$. The transient emission is shown for the case of triplet-triplet absorption (TTA) and singlet-triplet annihilation (STA). In both cases, the laser emission is short-lived. Also shown is the transient emission for a reduced triplet lifetime due to the presence of the plasmonic sink. It is found that the reduced triplet lifetime does not significantly affect the short time dynamics.

Figure 5: a) Steady-state triplet density for excitation with a square pulse of length $T_{\text {pulse }}=$ $20 \mathrm{~ns}$, shown as a function of repetition rate. The triplet density is also shown with the presence of the plasmonic sink resulting in an increase in the achievable repetition rate corresponding to the reduction in triplet lifetime. b) Relative increase in the laser threshold $R_{t h} / R_{t h 0}$, due to either STA or TTA, shown for several values of the pumping rate $(R)$. The last point of each curve corresponds to the maximum achievable laser repetition rate at the specified pump rate. It can be seen that the plasmonic limit 
corresponds to an increase in the achievable repetition rate of two orders of magnitude concomitant with the reduced triplet density.

Figure 6: a) Steady-state singlet and triplet densities calculated using Eq. (10), for plasmonic sink lattice spacings $L=100 \mathrm{~nm}, 250 \mathrm{~nm}$ and $400 \mathrm{~nm}$, normalized to their densities in the absence of quenching. Triplet and singlet diffusion lengths $L_{D, T}=250 \mathrm{~nm}$ and $L_{D, S}=20 \mathrm{~nm}$ were assumed. Note that for smaller lattice spacings, the triplet density is further reduced and the singlet density is left relatively unaffected until the lattice spacing reaches approximately $100 \mathrm{~nm}$. b) The optimal lattice spacing, $L_{\text {opt }}$, calculated with the objective of maximizing the relative change in triplet density and reducing the relative change in singlet density, is shown as a function of triplet diffusion length for $L_{D, S}=10 \mathrm{~nm}, 20 \mathrm{~nm}, 30 \mathrm{~nm}$ and $40 \mathrm{~nm}$. Note that for shorter singlet diffusion lengths, the lattice spacing can be made smaller, thus leading to a stronger reduction in the total number of triplets. c) The 2D steady-state singlet and triplet densities, normalized to their densities in the absence of quenching obtained for a lattice spacing $L=150 \mathrm{~nm}$ and disks of radius $\mathrm{r}=11 \mathrm{~nm}$. 
Graphical Table of Contents - Kéna-Cohen et al.

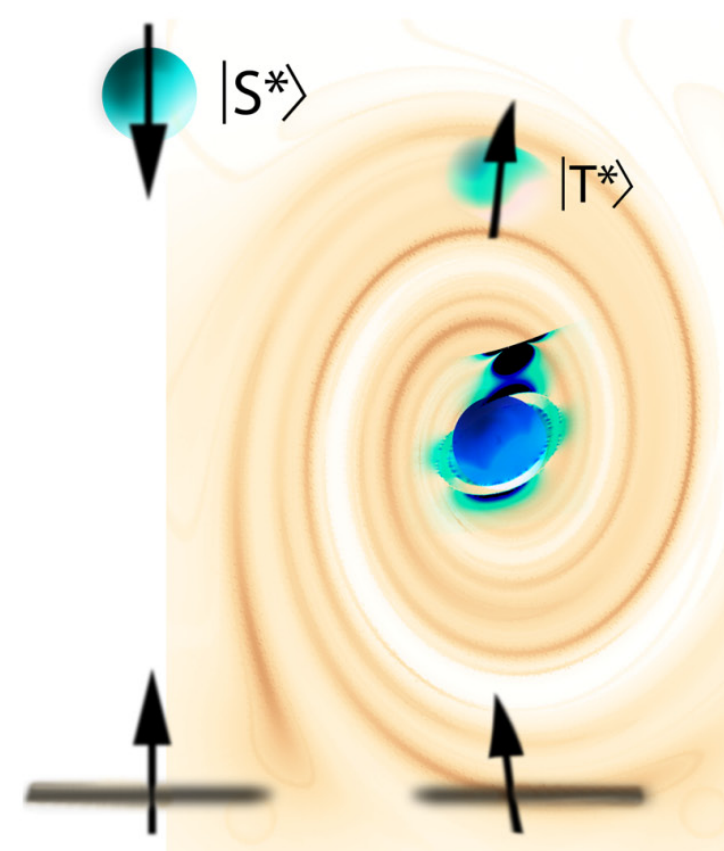

Triplets

Singlets

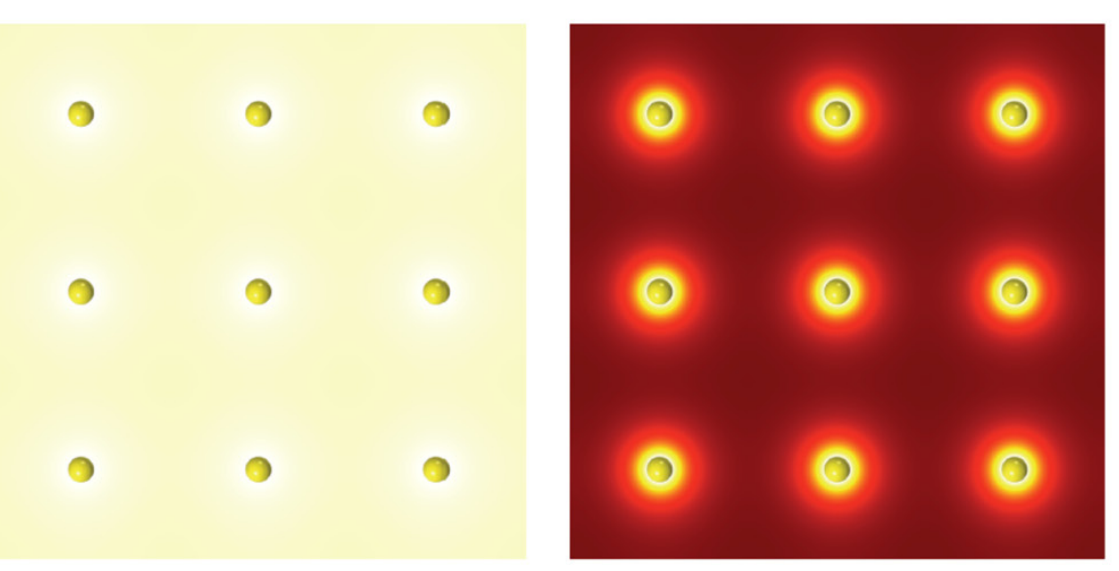




\section{Figure 1 - Kéna-Cohen et al.}
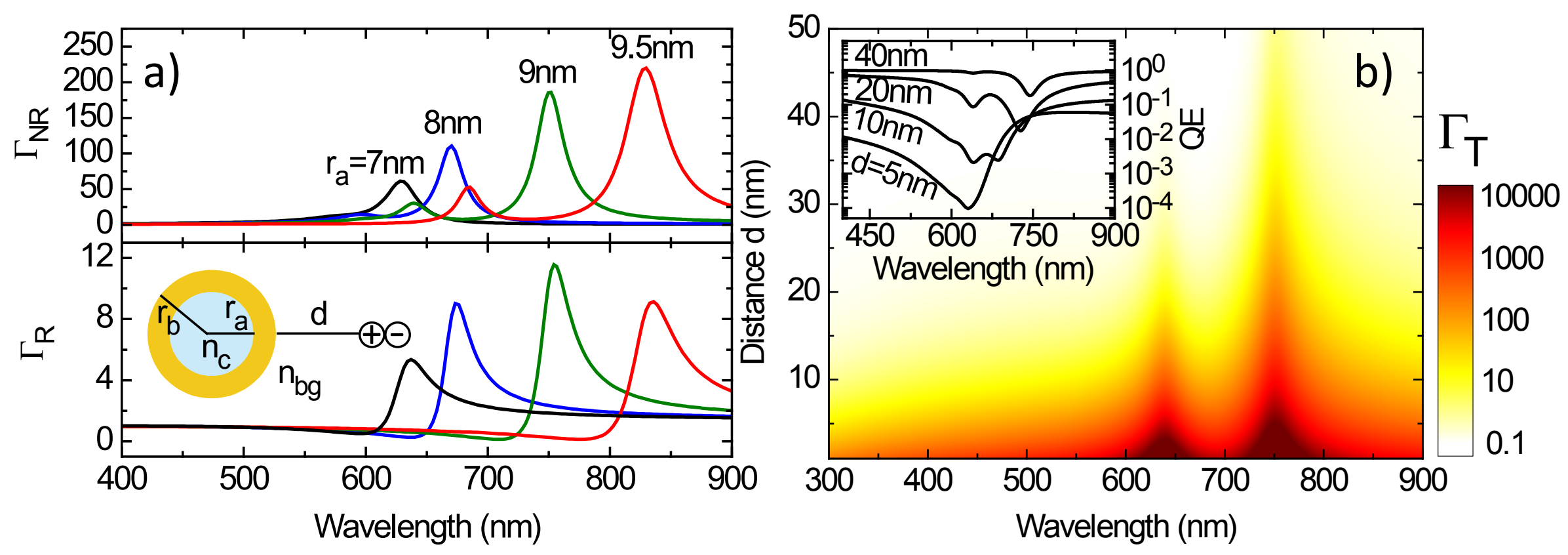
Figure 2 - Kéna-Cohen et al.

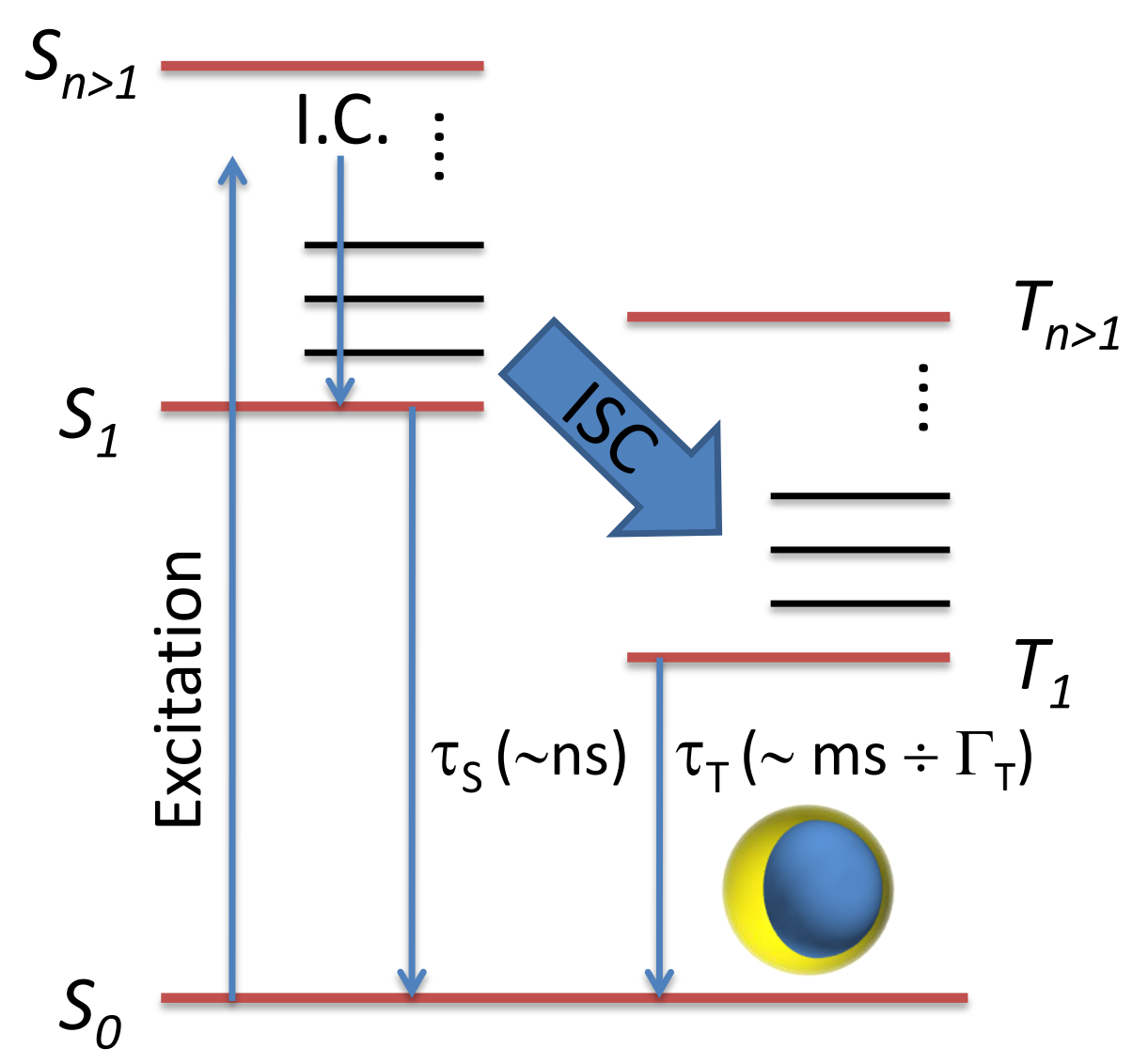


Figure 3 - Kéna-Cohen et al.
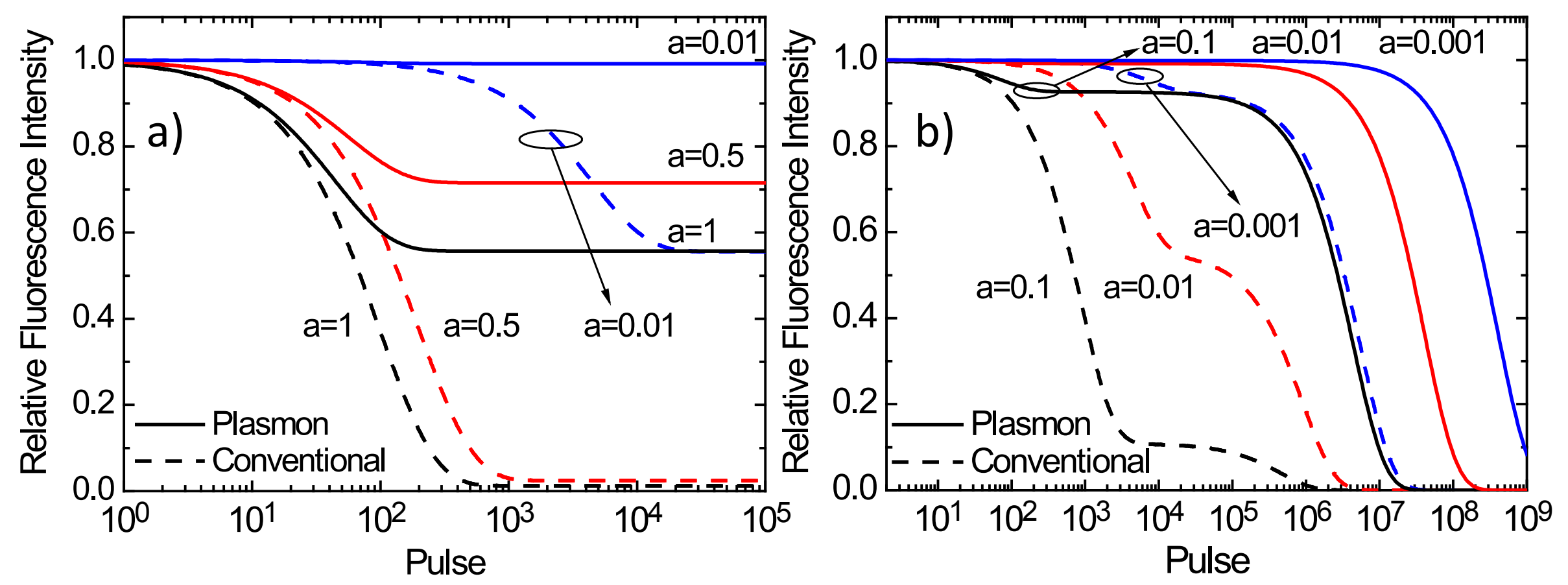
Figure 4 - Kéna-Cohen et al.

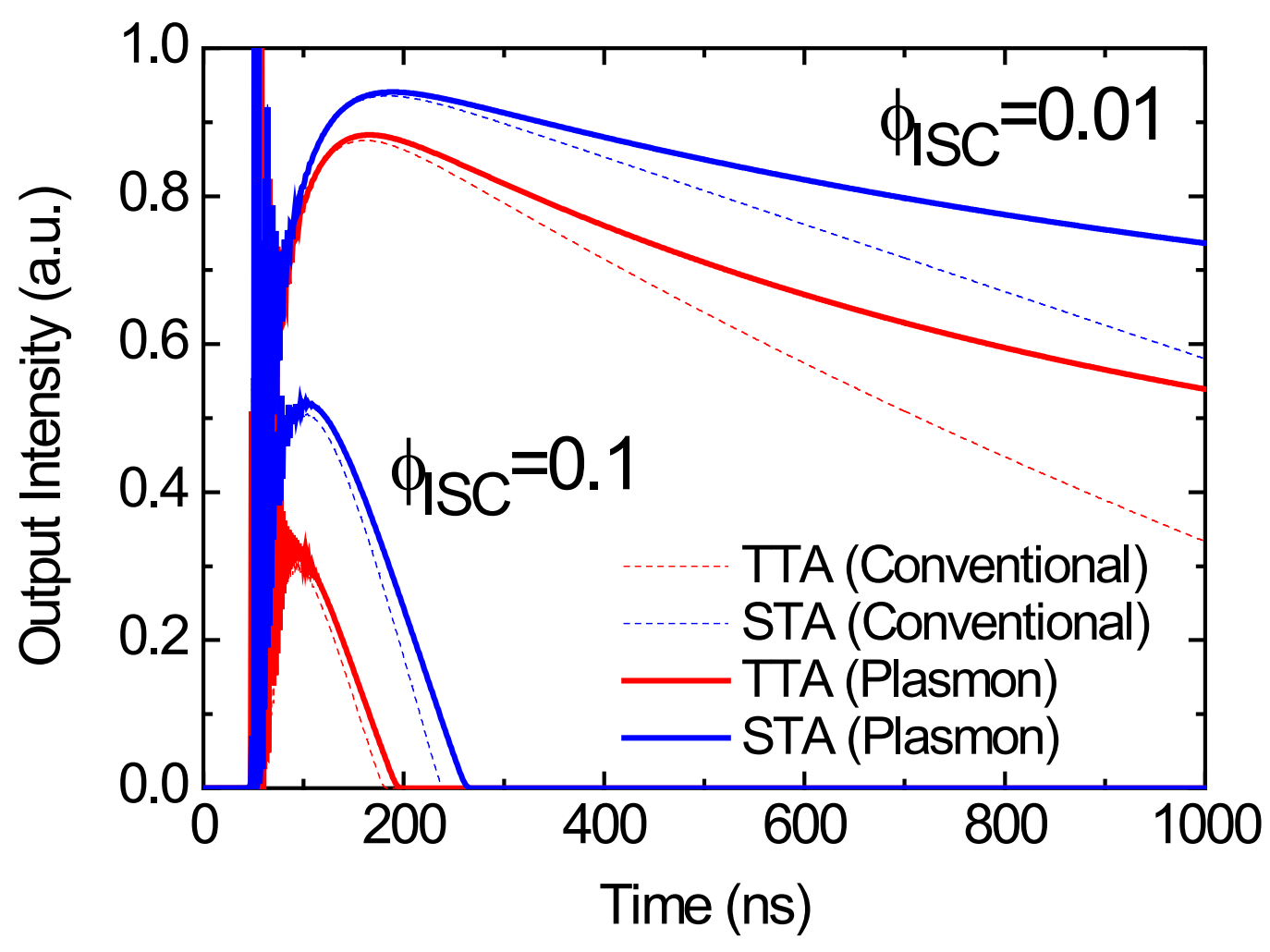




\section{Figure 5 - Kéna-Cohen et al.}
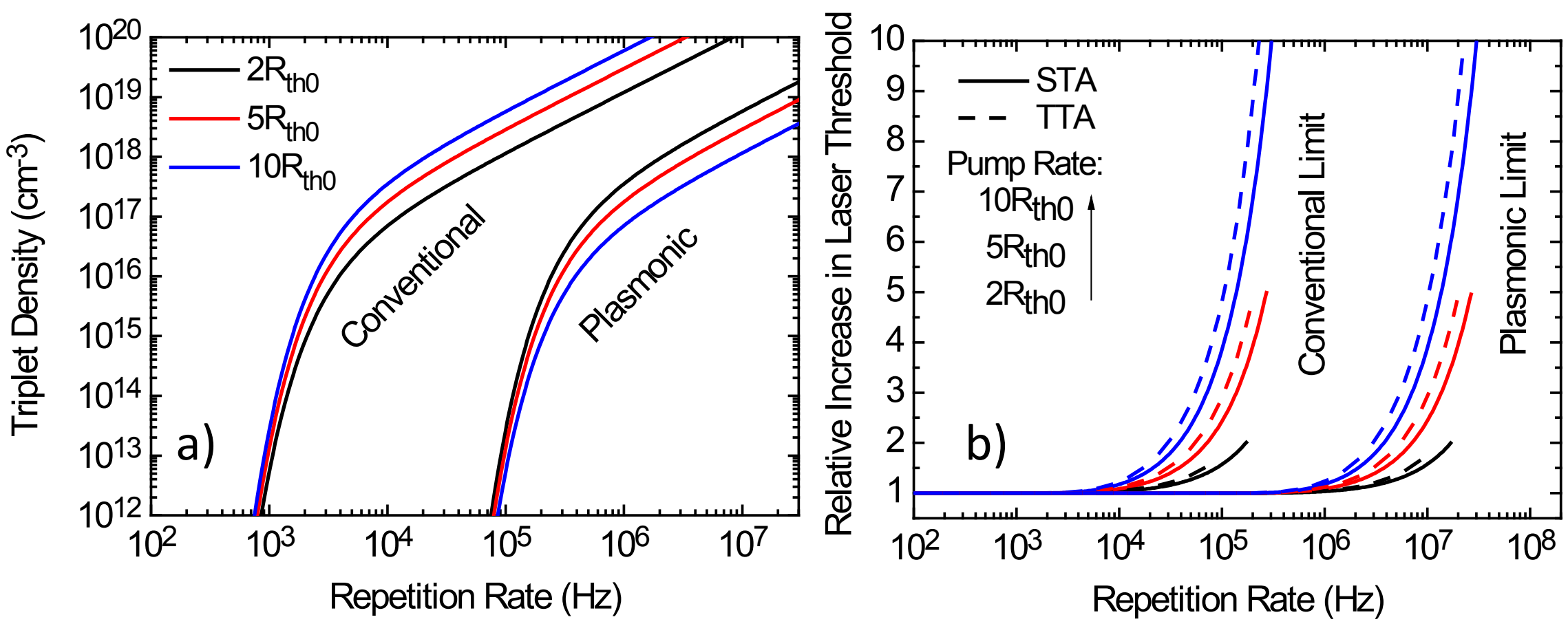
Figure 6 - Kéna-Cohen et al.
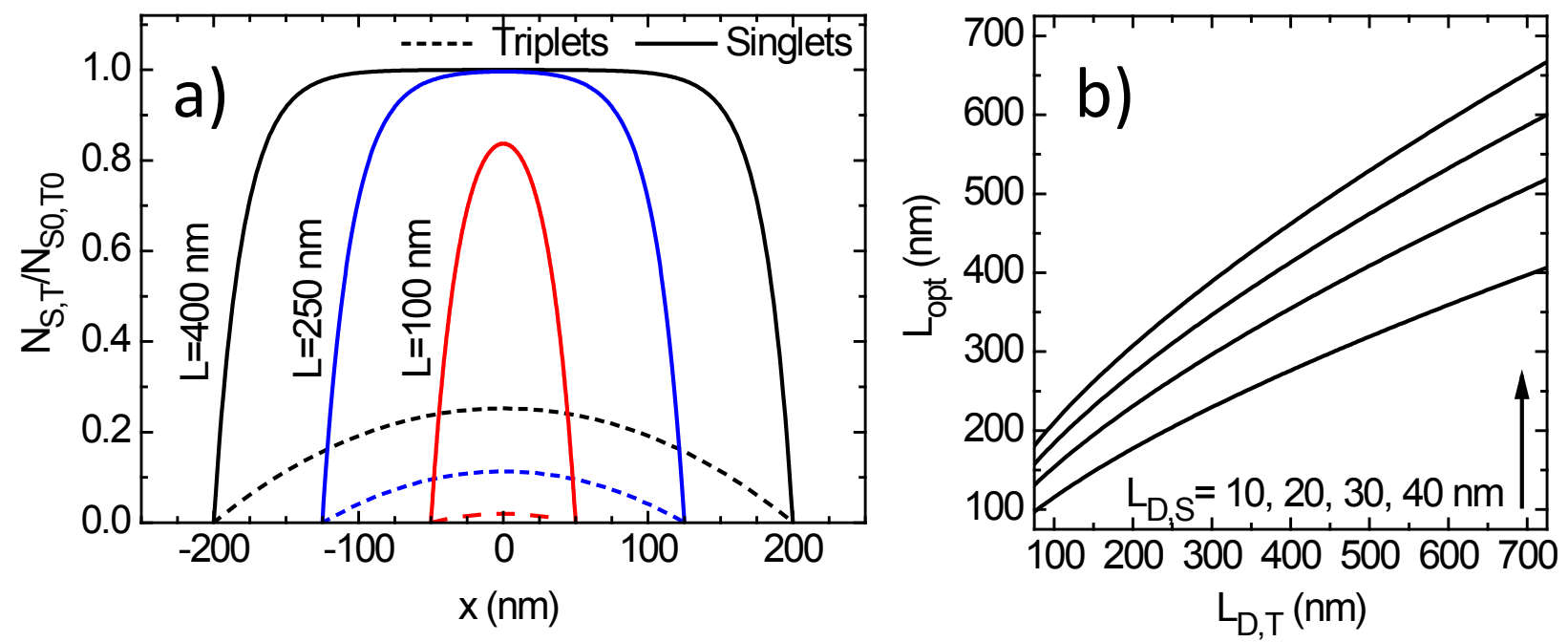

c)

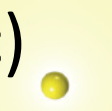

0

○

○

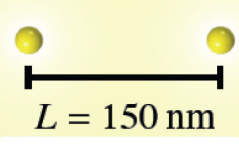

○

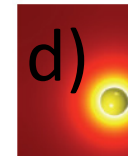

0 